\title{
Orientation Gradients in Rapidly Solidified Pure Aluminum Thin Films: Comparison of Experiments and Phase-Field Crystal Simulations
}

\author{
Paul Jreidini®, ${ }^{1}$ Tatu Pinomaa, ${ }^{2}$ Jörg M. K. Wiezorek, ${ }^{3}$ Joseph T. McKeown, ${ }^{4}$ Anssi Laukkanen $\odot,{ }^{2}$ and Nikolas Provatas ${ }^{1}$ \\ ${ }^{1}$ Department of Physics and Centre for the Physics of Materials, McGill University, \\ 3600 University Street, Montreal, QC, H3A 2T8, Canada \\ ${ }^{2}$ Integrated Computational Materials Engineering group, VTT Technical Research Centre of Finland Ltd, \\ Espoo, 02044, Finland \\ ${ }^{3}$ Department of Mechanical Engineering and Materials Science, University of Pittsburgh, \\ 636 Benedum Hall, 3700 O'Hara Street, Pittsburgh, Pennsylvania, 15261, USA \\ ${ }^{4}$ Materials Sciences Division, Physical and Life Science Directorate, Lawrence Livermore National Laboratory, \\ 7000 East Avenue, Livermore, California, 94551, USA
}

(Received 31 March 2021; revised 5 July 2021; accepted 19 October 2021; published 10 November 2021)

\begin{abstract}
Rapid solidification experiments on thin film aluminum samples reveal the presence of lattice orientation gradients within crystallizing grains. To study this phenomenon, a single-component phase-field crystal (PFC) model that captures the properties of solid, liquid, and vapor phases is proposed to model pure aluminium quantitatively. A coarse-grained amplitude representation of this model is used to simulate solidification in samples approaching micrometer scales. The simulations reproduce the experimentally observed orientation gradients within crystallizing grains when grown at experimentally relevant rapid quenches. We propose a causal connection between defect formation and orientation gradients.
\end{abstract}

DOI: 10.1103/PhysRevLett.127.205701

Modern industrial processes, including thermal spray coating deposition [1], certain welding techniques [2], and metal additive manufacturing [3], operate under rapid solidification conditions. Rapid solidification leads to drastically altered microstructures through selection of metastable phases, solute trapping kinetics [4-6], changes in solidification morphology [7], and reduction of dendrite primary arm spacing [7]. An overlooked key feature of these microstructures is the formation of various types of crystalline defects [8]. These include trapping of excess point vacancies [9], formation of high dislocation densities [10,11], high microstructural (type II-III) residual stresses [12], and lattice orientation gradients discussed below. As these crystalline defects critically affect materials' mechanical properties and performance, improving our understanding of their formation mechanisms leads to significant advances in the aforementioned processing techniques.

Among the various classes of crystalline defects, lattice orientation gradients formed during rapid solidification have been observed in numerous experimental systems yet remain poorly understood [13-16]. These defects consist of a gradual change in crystallographic orientation within a single grain, in contrast to the usual concept of an interface between

Published by the American Physical Society under the terms of the Creative Commons Attribution 4.0 International license. Further distribution of this work must maintain attribution to the author(s) and the published article's title, journal citation, and DOI. misoriented grains across which orientation changes abruptly. This observation is relatively novel in rapid solidification, and we are not aware of evidence for orientation gradients in slower solidification regimes. To our knowledge, no theoretical or numerical model has been proposed to study these orientation gradients [13], and this work aims elucidate the origins and characteristics of such defects. To that end, we first report on orientation gradients and related defect structures recently observed in rapid solidification experiments in pure aluminum. We then develop a model that produces comparable observations, and use simulations to rationalize the formation mechanisms of orientation gradients.

Establishing causal links between solidification conditions and resulting defected microstructures is challenging due to the highly transient processes over small observational timescales and length scales. Recent thin-film resolidification experiments have provided high quality information about emergent rapid solidification microstructures [17-21]. The approach is to use a pulsed laser beam to create an elliptical melt pool in a thin polycrystalline sample, and then let the sample resolidify $[19,20]$. We applied this procedure on a $100 \mathrm{~nm}$ thick polycrystalline sample of pure aluminium with a $50 \mathrm{~nm}$ thick amorphous $\mathrm{Si}_{3} \mathrm{~N}_{4}$ underlay. The as-solidified microstructure was imaged with a scanning/transmission electron microscope (S/TEM) on a spatial resolution of $5 \mathrm{~nm}$ and an angular resolution of $1^{\circ}$. The resulting inverse pole figure is shown in Fig. 1(a), where strong orientation gradients within grains are clearly visible and are highlighted with ellipses. 


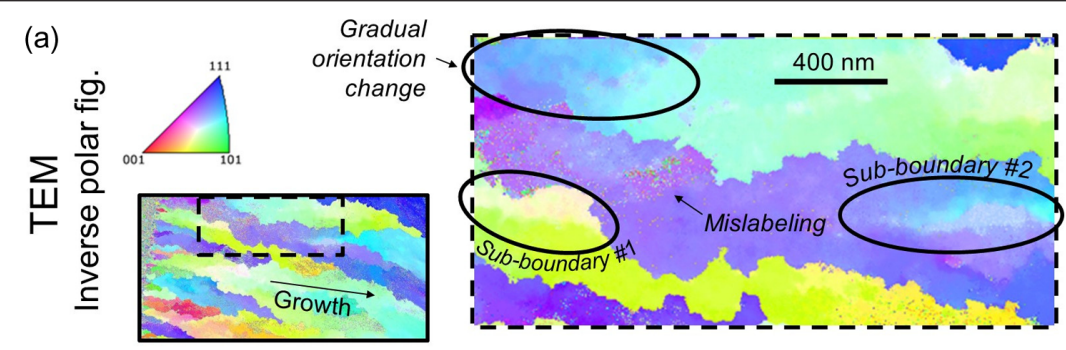

(b)

Development of a sub-boundary

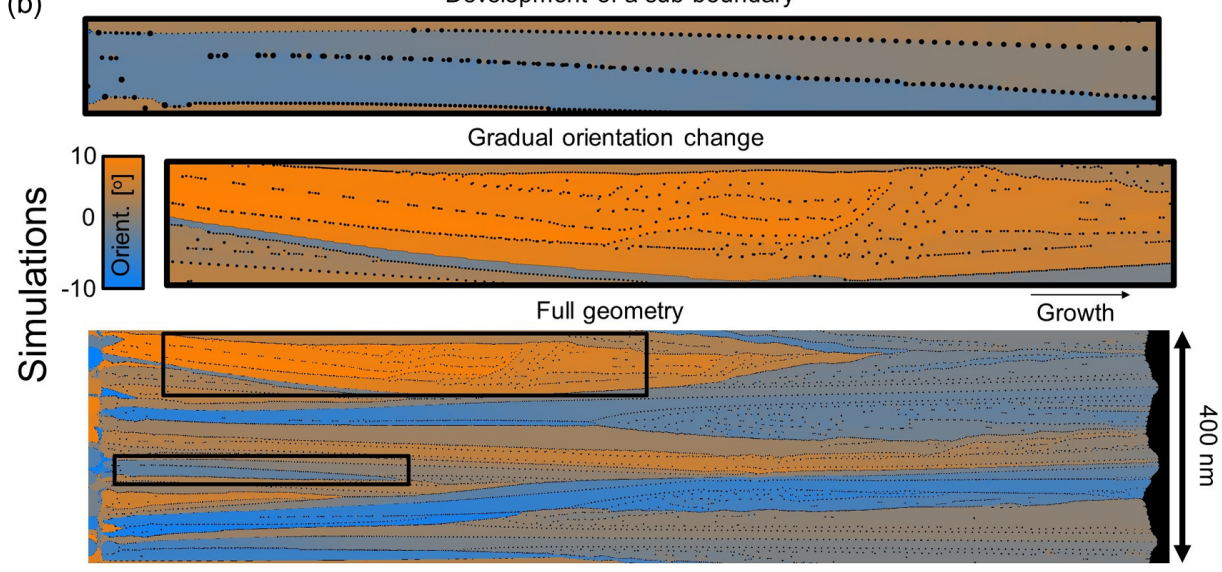

FIG. 1. Orientation distribution in rapidly solidified aluminum thin film, for (a) a laser thin film experiment as an inverse pole figure, and (b) in a phase-field crystal simulation. The color in (a) represents the crystallographic plane oriented in the plane of the image. The color in (b) represents a two-dimensional lattice orientation in the film plane, except for black, which represents noncrystalline areas such as liquid, voids, or dislocations. Orientation gradients are clearly visible within individual grains in (a) and (b). The overall growth directions are indicated with thin black arrows. Top frames in (b) are enlargements of the insets in the bottom frame.

These include gradual changes in orientation, and "subboundaries" which run approximately parallel to the growth direction and separate misoriented regions inside individual grains. The smooth orientation gradients inside grains are often accompanied by pixels in the image data where the local orientation varies abruptly, indicated as "mislabeling" in Fig. 1(a). A potential explanation for the mislabeling is high concentrations of crystalline defects. This hypothesis is explored later.

Since the experimental techniques lack the spatial resolution and temporal tracking needed to observe the formation of the orientation gradients in-situ at the nanometer scale, numerical approaches are required to complement the investigation of this phenomenon. This Letter proposes the use of a phase-field crystal (PFC) model [22], which can be considered an offshoot of classical density functional theory. Unlike traditional phase-field methods $[23,24]$, PFC is capable of self-consistently retaining lattice orientation and other atomic information while evolving microstructure dynamically on diffusive timescales. In this work, we expand on the PFC model proposed by Kocher et al. [25], which accounts for both liquid and vapor disordered phases as well as an ordered solid phase in a single component system. The existence of a vapor phase in the model is crucial under the assumption that extreme interface velocities can lead to trapping of vapor pockets, or "voids," in a manner analogous to solute trapping in alloys [6]. For an atomic density field $n(\mathbf{x})$, our model's free energy functional is written as

$$
\begin{aligned}
F[n]= & \int d \mathbf{x}\left(\sum_{l=2}^{4} \frac{1}{l} p_{l} n(\mathbf{x})^{l}+\sum_{l=2}^{4} \frac{1}{l} q_{l} n(\mathbf{x}) n_{m f}(\mathbf{x})^{l-1}\right) \\
& -\frac{1}{2} \iint d \mathbf{x}_{1} d \mathbf{x}_{2} C^{(2)}\left(\mathbf{x}_{1}-\mathbf{x}_{2}\right) n\left(\mathbf{x}_{\mathbf{1}}\right) n\left(\mathbf{x}_{\mathbf{2}}\right)
\end{aligned}
$$

where $n_{m f}(\mathbf{x})$ represents a Gaussian smoothing operation applied to the microscopic density $n(\mathbf{x})$, parameters $p_{l}$ and $q_{l}$ are temperature dependant and set the relative energies of the three accessible phases in thermodynamic equilibrium, and $C^{(2)}$ is a two-point correlation function that determines the lattice structure of the ordered solid phase. Here, we choose a $C^{(2)}$ yielding two-dimensional triangular lattice structure, represented in Fourier space by a single Gaussian peak [26,27]. While this PFC model could be simulated in three dimensions, practical computational constraints limit us to two. This does not significantly impact the comparison with the rapid solidification experiments, as those were done in thin-film geometry with thickness much smaller than length and width. Furthermore, while the choice of triangular lattice corresponds to a subset of possible 3D orientations of pure aluminum, our experimental observations [Fig. 1(a)] 


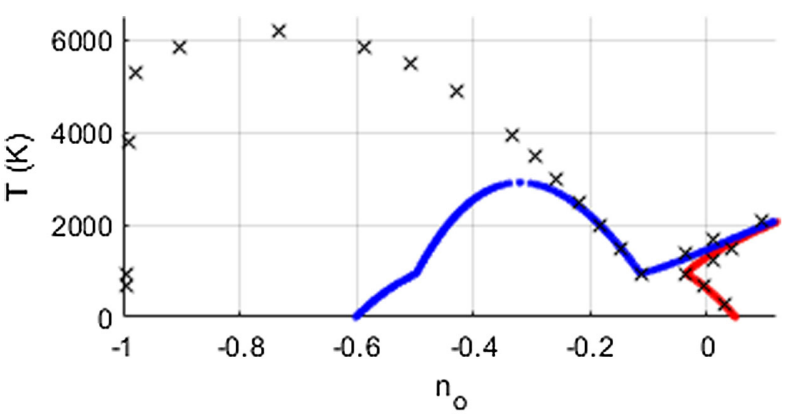

FIG. 2. Black crosses: thermodynamic phase diagram of pure aluminum [31]. Blue: Coexistence lines of disordered phases (liquid and vapor) of the proposed PFC model. Red: Coexistence line of the ordered (solid) phase of the model. The $y$ axis is temperature and the $x$ axis is average density (defined in the text).

suggest that qualitative morphology (including presence of orientation gradients) is independent of 3D orientation.

The parameters $p_{l}$ and $q_{l}$, and $C^{(2)}$, are chosen to obtain a thermodynamic phase diagram approaching that of pure aluminum in the vicinity of the triple point (see Supplemental Material 1 [28]) [29]. Figure 2 compares the PFC model's temperature-density phase diagram with that of pure aluminum. The temperature $T$ is in Kelvin, while the dimensionless average density $n_{o}$ is related to real density through $n_{o}=(\rho-\bar{\rho}) / \bar{\rho}$ where $\rho$ is the density and $\bar{\rho}$ is a reference density. The reference density (corresponding to rescaled density $n_{o}=0$ ) was chosen to be that of pure aluminum at solid coexistence for a temperature of $630 \mathrm{~K}$. The model's phase diagram deviates from pure aluminum's at densities below the critical point, due to the expanded nature of the free energy functional [22]. Further work is underway to expand the range of densities accessible in PFC models' phase diagrams [30]. As the present work focuses on solidification, accurate agreement with the solidus in the vicinity of the triple point was determined to be sufficient, and we choose initialization densities of our simulations taking into account the skewed lever rule resulting from the vapor coexistence's deviation (see Supplemental Material 3.1 [28]).

While PFC models evolve on diffusive timescales, their spatial resolution is limited to atomic length scales. To access larger simulation domains, our model is transformed into a complex order parameter phase-field model through the use of amplitude expansions [27,32]. This expands the atomic density as $n(\mathbf{x})=\bar{n}(\mathbf{x})+\sum_{j=1}^{3} A_{j}(\mathbf{x}) e^{i \mathbf{G}_{j} \cdot \mathbf{x}}+$ c.c. where $\bar{n}(\mathbf{x})$ is the long-wavelength density, $\mathbf{G}_{j}$ are the lowest-order reciprocal lattice vectors for a triangular lattice, and $A_{j}(\mathbf{x})$ are the complex amplitudes corresponding to each $\mathbf{G}_{j}$. Applying this expansion to Eq. (1) and following volumeaveraging techniques [27], we obtain an amplitude model defined by a free energy functional $F_{A}\left[A_{j}, \bar{n}\right]$. The evolution of the system is obtained by applying nonconserved dissipative dynamics on $A_{j}(\mathbf{x})$ and conserved dissipative dynamics on $\bar{n}(\mathbf{x})$ (see Supplemental Material 2 [28]) [27].
The amplitude model was simulated in a comoving reference frame with an initial condition consisting of grains of random orientation up to $10^{\circ}$ from a reference orientation (see Supplemental Material 3.2 [28]). The average temperature of the system was set to $T=805 \mathrm{~K}$, a significant quench below pure aluminum's melting point of approximately $933 \mathrm{~K}$. A frozen temperature gradient of $G=5 \times 10^{7} \mathrm{~K} \mathrm{~m}^{-1}$ was applied along the solid-liquid interface. As experiments presented in this work did not include temperature measurements, simulation temperature conditions were chosen based on conditions for similar rapidly solidified thin-film samples [24]. Average density of the initial melt was $n_{o}=-0.040$. The total width and length of the simulated sample were approximately 400 and $2000 \mathrm{~nm}$, respectively. These scales where chosen to be accessible numerically while still capturing the pertinent nanoscale phenomena emerging from rapid solidification. We expect essential features discussed subsequently to be comparable to experimental results despite the difference in length scales.

To compare with experimentally observed orientation gradients, the lattice orientations in simulated solid grains were calculated from the atomic displacement field extracted from the complex amplitude fields $A_{j}$ [33]. Figure 1(b) shows a collage of lattice orientations in a postsolidified simulated sample. These are reconstructed from snapshots of the comoving frame as it advanced into the melt, and are compared to similar microstructure observed in experiments [Fig. 1(a)]. We observe orientation gradients in simulated grains, in some cases on the order of $10^{\circ}$ over $1000 \mathrm{~nm}$. Figure 3 shows zoomed-in sections of experimental and simulated samples, plotting the change in orientation in individual grains as a function of distance. Simulated and experimental orientation gradient magnitudes are in fair agreement considering the aforementioned model limitations.

Solid bulks shown in Figs. 1(b) and 3(b) reveal various defect types. In the simulation results displayed, larger black areas within and between solid grains, where black corresponds to areas of low absolute value of the fields $A_{j}$, are found to be at densities approaching that of the vapor phase, while the smaller such black areas tend to be closer in density to the solid. A distinction can be made between these different sized defects: The larger, low-density areas are voids (vapor pockets) formed due to density trapping at high interface velocities, while the smaller defects are dislocations. However, this distinction is not clear cut, as many of the voids are found to exhibit dipolar strain fields indicating a topological nature equivalent to dislocations. Additionally, close observation of the system's time evolution reveals that some voids, particularly those along grain boundaries, can shrink into dislocations as density diffuses, and vice versa. A plausible cause for this interchangeability is the pointlike nature of dislocations 
(a)

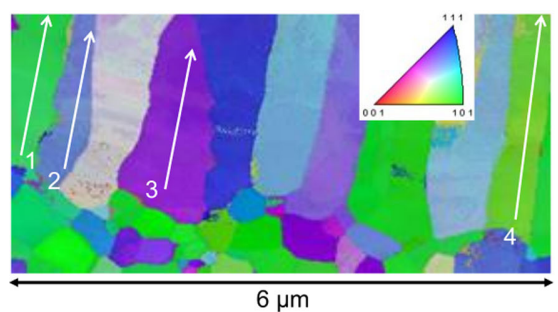

(c)

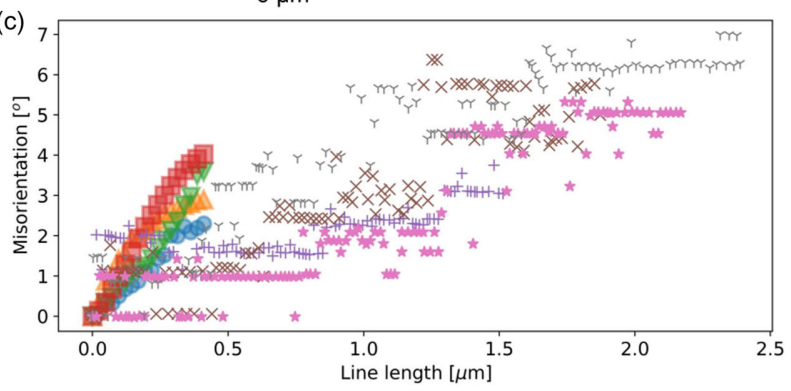

(b)

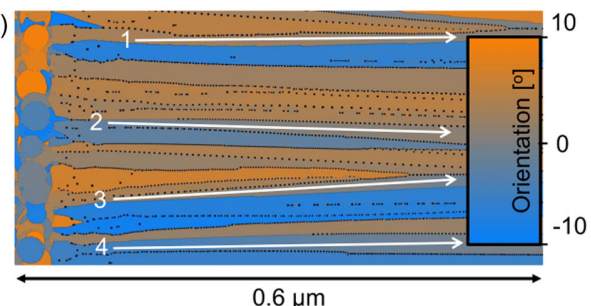

$0.6 \mu \mathrm{m}$

$+\# 1$

\#2 Experiments

\# \#3

Ү \#4

- \#1

A $\# 2$ Simulations

\#4

FIG. 3. Orientation distributions within single grains in (a) the laser thin film experiment and (b) the phase-field crystal simulation are shown, and (c) misorientation along labeled lines are plotted. Color maps in top images are the same as in Fig. 1. The orientation gradients calculated along the labeled lines are found to lie in the range $2.4-4.3^{\circ} \mu \mathrm{m}^{-1}$ for the experiments and $7.9-10^{\circ} \mu \mathrm{m}^{-1}$ for the simulations.

in two-dimensional systems. Alternatively, this could be explained by the diffusive merger of dislocations to form voids, as suggested by previous literature [34]. Figures 4(a) and 4(b) showcase simulated defect structures spanning this range of behaviors (see also Supplemental Material 4 [28]). Though experimental results tended to be of too low resolution to explicitly observe dislocations, it is plausible that the "mislabeled pixels" seen in the S/TEM inverse pole
Fig. 1(a) are likely due to algorithmic misclassification of orientation caused by high densities of dislocations. Further, some TEM images [shown in Fig. 4(c)] displayed rows of large-scale defects with dipolar strain fields. If these defects were dislocations, their interspacing of $70-100 \mathrm{~nm}$ would correspond to intergrain misorientation less than $0.3^{\circ}$, much smaller than the common misorientations observed in our samples. We thus propose they could (a)

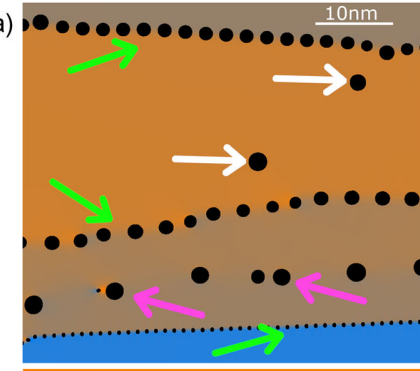

(b)

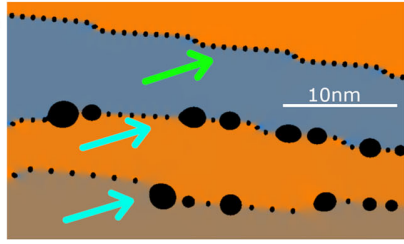

(c)

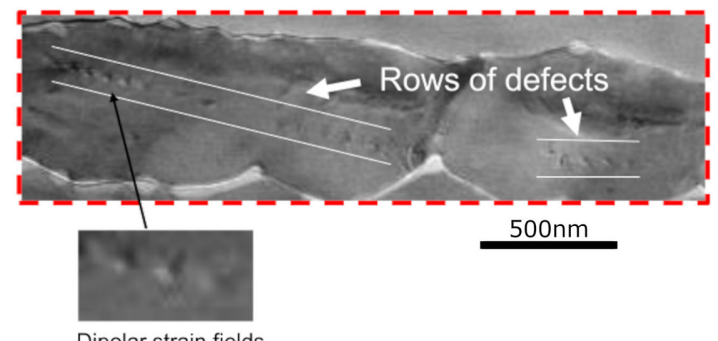

Dipolar strain fields

(d)

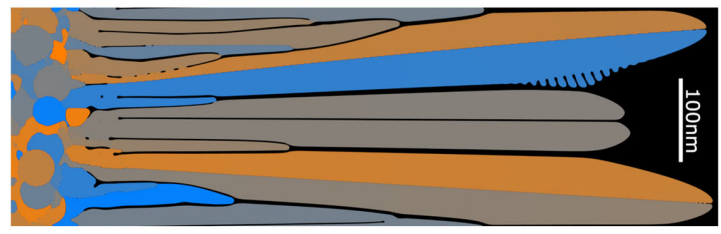

FIG. 4. (a) and (b) Various defect types observed in enlarged subsections of the simulated rapidly solidified solid. White arrows show isolated voids within a single grain. Green arrows show rows of dislocations, each separating grains of differing orientations. Though these marked rows exhibit regular spacing and dipolar strain fields, the extent of ordering of the dislocation cores differs between rows. Further, the green-marked dislocation row in (b) exhibits steplike structure, likely a result of lattice misfit accommodation [38-40]. Pink arrows show a row of what appears to be defects of an intermediate nature: unevenly spaced and sized, some exhibiting a dipolar strain field, and with inconsistent internal density. These emerge within a single grain that splits into two slightly different orientations as the solidification proceeds. The cyan arrows show defect arrays that consist of regularly spaced dislocations and voids, observed to interchangeably evolve diffusely in time as mentioned in the text. (c) Defect rows observed in TEM imaging of an experimental sample. (d) Collage of simulated polycrystalline solidification of $\mathrm{Al}$ at low cooling rate corresponding to quench temperature $T=875 \mathrm{~K}$. Color map is the same as in Fig. 1(b). 
be voids. Similar voids have previously been observed in rapidly solidified samples [35-37].

During dynamical simulations of rapid solidification, lattice orientation in solid grains is observed to evolve only within a few diffusion lengths of the solidifying front. Further behind this interface, the orientation appears "locked in" until the relevant part of the grain moves out of the comoving frame's limit. The formation of orientation gradients is always accompanied by specific dislocation behavior at the solid-liquid interface. Namely, when a growing grain is observed to undergo a gradual rotation, the spacing of observed dislocations forming at the boundary between this rotating grain and its neighbors is found to vary steadily as the rotation proceeds. Certain grains exhibit more chaotic behavior, splitting into multiple growing tips of slightly different orientations instead of rotating as a single lattice. This can be compared to the experimental images of Fig. 1(a), where such splitting is manifested as subboundaries often preceded by the mislabeled pixels mentioned above. Again, this simulated behavior is observed to be closely tied to the formation of dislocations at the interface, usually being preceded by sporadic trapped voids that exhibit a weak dipolar strain field surrounding them. These observations collectively indicate that the formation of orientation gradients within the bulk is linked to dislocations developing at the solidifying front, and the formation of these dislocations is often itself tied to the appearance of series of voids caused by density trapping that gradually acquire a topological nature.

To better illustrate the relation between defect formation and orientation gradients that emerge at rapid solidification rates, we also conducted simulations at higher temperature $T=875 \mathrm{~K}$, in the limit of no frozen temperature gradient $(G=0)$ and with initial melt density $n_{o}=-0.045$. Here, lower driving forces for solidification lead to lower interface velocities. Figure 4(d) shows a collage of the solidified material's lattice orientations. Unlike the case for rapidly solidified $\mathrm{Al}$ discussed above, here the intergrain boundaries tended to remain "wet," consisting of metastable fluid, with fewer cases of "dry" boundaries containing dislocation arrays. The grains also displayed dendrite arm formation, as expected for the lower driving forces involved. We observed in this and other simulations at low solidification rates that orientation gradients in grains were much weaker and rarer than in the case of the rapid solidification results reported above. Specifically, at low solidification rates, noticeable orientation gradients were only observed in grains that shared dry grain boundaries, since wet boundaries do not effectively communicate strains between newly forming solid lattices. These results support our hypothesis that the experimentally observed orientation gradients are connected to the formation of dislocation arrays at grain boundaries starting from instabilities at the solidifying front. This also emphasizes that the appearance of orientation gradients is a phenomenon intrinsically tied to high interface velocities prevalent in rapid solidification regimes.

In summary, this work reports new observations of orientation gradients in pure aluminum thin film resolidification experiments and in simulations of a novel 3-phase single-component PFC model of aluminum. Simulated orientation gradients are in fair agreement with experiments. The likely presence of defects in experimental images, indicated by mislabeled pixels in inverse pole figures as well as the observed presence of defect arrays, agrees with simulation results and suggests the importance of dislocations and voids in mediating the formation of orientation gradients within individual grains. Future work will benefit from accurate measurement of experimental thermal profiles, as well as higher resolution experimental imaging techniques capable of examining defects at length scales comparable to that of the simulations. PFC modeling improvements, including three-dimensional simulations, dynamically co-evolving temperature [30], expanded phase diagram matching, and better matching of elastic and interfacial energies, are expected to further improve agreement between simulation and experimental observations presented above.

N. P. acknowledges the National Science and Engineering Research Council of Canada (NSERC) and the Canada Research Chairs (CRC) Program for support. T. P. and A. L. acknowledge the Academy of Finland, HEADFORE project, Grant No. 333226, for support. J. M. K. W. acknowledges the National Science Foundation under Grant No. DMR 1607922 for support. J. T. M. was funded under the auspices of the U.S. Department of Energy under Contract No. DE-AC52-07NA27344.

[1] E. J. Lavernia and T. S. Srivatsan, The rapid solidification processing of materials: Science, principles, technology, advances, and applications, J. Mater. Sci. 45, 287 (2010).

[2] S. A. David, S. S. Babu, and J. M. Vitek, Welding: Solidification and microstructure, JOM 55, 14 (2003).

[3] T. DebRoy, H. L. Wei, J. S. Zuback, T. Mukherjee, J. W. Elmer, J. O. Milewski, A. M. Beese, A. Wilson-Heid, A. De, and W. Zhang, Additive manufacturing of metallic components-process, structure and properties, Prog. Mater. Sci. 92, 112 (2018).

[4] J. W. Cahn, W. C. Carter, and W. C. Johnson, The Selected Works of John W. Cahn (Wiley-TMS, Pittsburgh, 1998).

[5] M. J. Aziz, Interface attachment kinetics in alloy solidification, Metall. Mater. Trans. A 27, 671 (1996).

[6] T. Pinomaa, A. Laukkanen, and N. Provatas, Solute trapping in rapid solidification, MRS Bull. 45, 910 (2020).

[7] R. Trivedi and W. Kurz, Solidification microstructures: A conceptual approach, Acta Metall. Mater. 42, 15 (1994).

[8] R. W. Balluffi, S. M. Allen, and W. C. Carter, Kinetics of Materials (John Wiley \& Sons, New York, 2005). 
[9] H. Y. Zhang, F. Liu, Y. Yang, and D. Y. Sun, The molecular dynamics study of vacancy formation during solidification of pure metals, Sci. Rep. 7, 10241 (2017).

[10] Y. M. Wang, T. Voisin, J. T. McKeown, J. Ye, N. P. Calta, Z. Li, Z. Zeng, Y. Zhang, W. Chen, T. T. Roehling et al., Additively manufactured hierarchical stainless steels with high strength and ductility, Nat. Mater. 17, 63 (2018).

[11] G. Wang, H. Ouyang, C. Fan, Q. Guo, Z. Li, W. Yan, and Z. $\mathrm{Li}$, The origin of high-density dislocations in additively manufactured metals, Mater. Res. Lett. 8, 283 (2020).

[12] W. Chen, T. Voisin, Y. Zhang, J.-B. Florien, C. M. Spadaccini, D. L. McDowell, Ting Zhu, and Y. M. Wang, Microscale residual stresses in additively manufactured stainless steel, Nat. Commun. 10, 4338 (2019).

[13] A. T. Polonsky, W. C. Lenthe, M. P. Echlin, V. Livescu, G. T. Gray III, and T. M. Pollock, Solidification-driven orientation gradients in additively manufactured stainless steel, Acta Mater. 183, 249 (2020).

[14] Z. Wang, T. A. Palmer, and A. M. Beese, Effect of processing parameters on microstructure and tensile properties of austenitic stainless steel 3041 made by directed energy deposition additive manufacturing, Acta Mater. 110, 226 (2016).

[15] A. Riemer, S. Leuders, M. Thöne, H. A. Richard, T. Tröster, and T. Niendorf, On the fatigue crack growth behavior in 3161 stainless steel manufactured by selective laser melting, Eng. Fract. Mech. 120, 15 (2014).

[16] Y. Lin, S. Mao, Z. Yan, Y. Zhang, and L. Wang, The enhanced microhardness in a rapidly solidified al alloy, Mater. Sci. Eng. A 692, 182 (2017).

[17] R. Zhong, Microstructural study of laser-induced rapid lateral solidification of metal thin films, Ph.D. thesis, University of Pittsburgh, 2010.

[18] A. Kulovits, J. M. K. Wiezorek, T. LaGrange, B. W. Reed, and G. H. Campbell, Revealing the transient states of rapid solidification in aluminum thin films using ultrafast in situ transmission electron microscopy, Philos. Mag. Lett. 91, 287 (2011).

[19] J. T. McKeown, K. Zweiacker, C. Liu, D. R. Coughlin, A. J. Clarke, J. K. Baldwin, J. W. Gibbs, J. D. Roehling, S. D. Imhoff, P. J. Gibbs et al., Time-resolved in situ measurements during rapid alloy solidification: Experimental insight for additive manufacturing, JOM 68, 985 (2016).

[20] K. W. Zweiacker, Can Liu, M. A. Gordillo, J. T. McKeown, G. H. Campbell, and J. M. K. Wiezorek, Composition and automated crystal orientation mapping of rapid solidification products in hypoeutectic al-4 at. $\% \mathrm{cu}$ alloys, Acta Mater. 145, 71 (2018).

[21] V. Bathula, C. Liu, K. Zweiacker, J. McKeown, and J. M. K. Wiezorek, Interface velocity dependent solute trapping and phase selection during rapid solidification of laser melted hypo-eutectic al-11at.\%cu alloy, Acta Mater. 195, 341 (2020).

[22] K. R. Elder, N. Provatas, J. Berry, P. Stefanovic, and M. Grant, Phase-field crystal modeling and classical density functional theory of freezing, Phys. Rev. B 75, 064107 (2007).
[23] W. J. Boettinger, J. A. Warren, C. Beckermann, and A. Karma, Phase-field simulation of solidification, Annu. Rev. Mater. Res. 32, 163 (2002).

[24] T. Pinomaa, J. T. McKeown, J. M. K. Wiezorek, N. Provatas, A. Laukkanen, and T. Suhonen, Phase field modeling of rapid resolidification of $\mathrm{Al}-\mathrm{Cu}$ thin films, J. Cryst. Growth 532, 125418 (2020).

[25] G. Kocher and N. Provatas, New Density Functional Approach for Solid-Liquid-Vapor Transitions in Pure Materials, Phys. Rev. Lett. 114, 155501 (2015).

[26] M. Greenwood, N. Provatas, and J. Rottler, Free Energy Functionals for Efficient Phase Field Crystal Modeling of Structural Phase Transformations, Phys. Rev. Lett. 105, 045702 (2010).

[27] N. Ofori-Opoku, J. Stolle, Z.-F. Huang, and N. Provatas, Complex order parameter phase-field models derived from structural phase-field-crystal models, Phys. Rev. B 88, 104106 (2013).

[28] See Supplemental Material at http://link.aps.org/supplemental/ 10.1103/PhysRevLett.127.205701 for additional modeling and simulation details.

[29] P. Jreidini, Ph.D. thesis, McGill University, (2022) (unpublished doctoral dissertation).

[30] G. Kocher and N. Provatas, Thermodensity coupling in phase-field-crystal-type models for the study of rapid crystallization, Phys. Rev. Mater. 3, 053804 (2019).

[31] I. V. Lomonosov, Multi-phase equation of state for aluminum, Laser Part. Beams 25, 567 (2007).

[32] B. P. Athreya, N. Goldenfeld, and J. A. Dantzig, Renormalization-group theory for the phase-field crystal equation, Phys. Rev. E 74, 011601 (2006).

[33] V. Heinonen, C. V. Achim, J. M. Kosterlitz, S.-C. Ying, J. Lowengrub, and T. Ala-Nissila, Consistent Hydrodynamics for Phase Field Crystals, Phys. Rev. Lett. 116, 024303 (2016).

[34] A. M. Cuitiño and M. Ortiz, Ductile fracture by vacancy condensation in f.c.c. single crystals, Acta Mater. 44, 427 (1996).

[35] A. Michalcová, D. Vojtěch, J. Čížek, I. Procházka, J. Drahokoupil, and P. Novák, Microstructure characterization of rapidly solidified Al-Fe-Cr-Ce alloy by positron annihilation spectroscopy, J. Alloys Compd. 509, 3211 (2011).

[36] M. C. Pandey, J. Wadsworth, and A. K. Mukherjee, Cavitation study on ingot and powder metallurgically processes superplastic Al-Li alloys, Mater. Sci. Eng. 78, 115 (1986).

[37] A. Rukwied, A. W. Ruff, and W. A. Willard, Study of the cellular solidification structure in a continuously cast high purity copper, Metall. Mater. Trans. B 2, 2105 (1971).

[38] T. Furuhara, J. M. Howe, and H. I. Aaronson, Interphase boundary structures of intragranular proeutectoid $\alpha$ plates in a hypoeutectoid TiCr alloy, Acta Metall. Mater. 39, 2873 (1991).

[39] K. Fujiwara, H. Matsunoshita, Y. Sasai, K. Kishida, and H. Inui, Effects of ternary additions on the microstructure and thermal stability of directionally-solidified MoSi2/Mo5Si3 eutectic composites, Intermetallics 52, 72 (2014).

[40] J.-Y. Zhang, Y. Gao, Y. Wang, and W.-Z. Zhang, A generalized O-element approach for analyzing interface structures, Acta Mater. 165, 508 (2019). 